\title{
Synthesis and NMR-spectroscopic characterization of diastereomeric bicyclo[4.2.0]octane-2,7-diones
}

\author{
Kerstin Schmidt and Paul Margaretha* \\ Department of Chemistry, University of Hamburg, D-20146 Hamburg, Germany \\ E-mail: Paul.Margaretha@,chemie.uni-hamburg.de
}

Dedicated to Dr. Arlette Solladié-Cavallo on the occasion of her birthday

\begin{abstract}
Irradiation $(\lambda=350 \mathrm{~nm})$ of cyclohexenones 4 in the presence of 1,1-dimethoxyethylene (5) affords mixtures of diastereomeric 7,7-dimethoxybicyclo[4.2.0]octan-2-ones 6 and 7. Careful hydrolysis of these acetals with acidic $\mathrm{SiO}_{2}$ affords the title compounds 8 and $\mathbf{9}$ quantitatively without any epimerization at $\mathrm{C}(1)$. These bicyclic diketones were fully characterized by ${ }^{1} \mathrm{H}-$ and ${ }^{13} \mathrm{C}-\mathrm{NMR}$ spectroscopy.
\end{abstract}

Keywords: Enone photocycloaddition, acetal hydrolysis, trans-fused cyclobutanones

\section{Introduction}

Bicyclo[4.2.0]octanes ares easily accessible by [2+2]-cycloaddition of a cyclohexene moiety to an alkene. ${ }^{1,2}$ Regarding the two diastereomeric parent bicycloalkanes $\mathbf{1 a}$ and $\mathbf{2 a}$, the trans-fused stereoisomer $1 \mathbf{a}$ is expected to be more strained by about $25 \mathrm{~kJ} / \mathrm{mol}$ than its cis-fused counterpart 2a. ${ }^{3}$ Insertion of a trigonal planar $\mathrm{C}$-atom into the six-membered ring leads to a slight increase in strain energy for both diastereomers and it is therefore not surprising, that trans-fused bicyclo[4.2.0] octan-2-ones, e.g. 1b, epimerize readily to the thermodynamically more stable cisfused diastereomer $\mathbf{2 b}$ on treatment with base or acid. ${ }^{4}$ Incorporation of an additional carbonyl group, now into the four-membered ring, should not influence the relative stability of the resulting bicyclo[4.2.0] octane-2,7-diones $\mathbf{1 c}$ and $\mathbf{2 c}$, as the strain energy of cyclobutanone is very similar to that of cyclobutane itself. ${ }^{5}$ Interestingly, up to now only one such trans-fused (polycyclic) cyclobutanone $\mathbf{3}$, resulting from [2+2]-photocycloaddition of a steroidal cyclohexenone to a ketene acetal, followed by hydrolysis of the acetal function and then characterized mainly by IR-spectroscopy, has been reported in the literature (Figure 1). ${ }^{6}$ Here we report on the synthesis and full NMR-spectroscopic characterization of such pairs of bicyclic title compounds. 


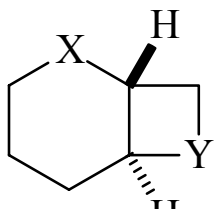

$\mathrm{H}$

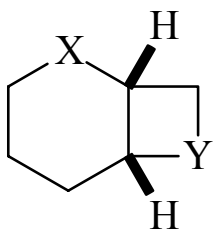

2

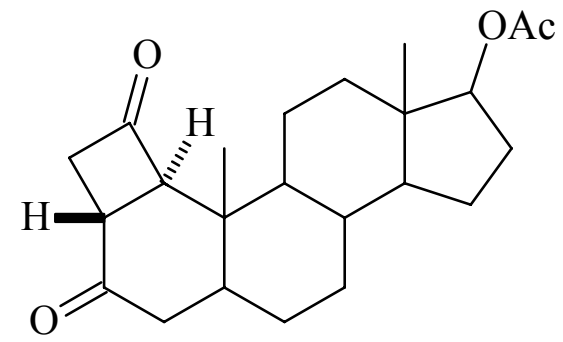

a: $\mathrm{X}=\mathrm{Y}=\mathrm{CH}_{2}$

b: $\mathrm{X}=\mathrm{CO}, \mathrm{Y}=\mathrm{CH}_{2}$

c: $\mathrm{X}=\mathrm{Y}=\mathrm{CO}$

Figure 1

\section{Results and Discussion}

Irradiation $(350 \mathrm{~nm})$ of cyclohexenones 4 in benzene in the presence of a tenfold molar excess of 1,1-dimethoxyethylene (5) affords regioselectively diastereomeric mixtures of 7,7dimethoxybicyclo[4.2.0] octan-2-ones $\mathbf{6}$ and 7. The excess of added alkene prevents photodimerization of $\mathbf{4}$. For 5,5-dimethylcyclohex-2-enone (4a) the product ratio $\mathbf{6 a} / \mathbf{7 a}$ is $3: 2$, and for 4,4-dimethylcyclohex-2-enone (4b) the ratio $\mathbf{6 b} / \mathbf{7 b}$ is $5: 2$, respectively. Differentiation between the (major) trans- and (minor) cis-fused bicyclooctanones $\mathbf{6}$ and $\mathbf{7}$ is straightforward by means of the magnitude of the vicinal coupling constant for the bridgehead H-atoms $\left({ }^{3} J \approx 13-14\right.$ $\mathrm{Hz}$ for $\mathbf{6 a} / \mathbf{7 a}$, and $\approx 10-11 \mathrm{~Hz}$ for $\mathbf{6 b} / \mathbf{7 b}$ ), and also by the ease of epimerization of the former to the latter diastereomers in the presence of either base or acid. Hydrolysis of the acetal function can be achieved quantitatively under mild aqueous acidic conditions, either using a two phase ( $10 \%$ aq. $\mathrm{HCl} / \mathrm{CH}_{2} \mathrm{Cl}_{2}$ ) system, ${ }^{7}$ or $\mathrm{SiO}_{2}$ pretreated with $15 \%$ aq. $\mathrm{H}_{2} \mathrm{SO}_{4}$ in $\mathrm{CH}_{2} \mathrm{Cl}_{2}{ }^{8}$. Whereas the first method is accompanied by rapid epimerization of the trans-fused diketones to the cisfused diastereomers, hydrolysis of the product mixtures $6 \mathbf{a} / 7 \mathbf{a}$ or $6 \mathbf{b} / 7 \mathbf{b}$ according to the second method for $4 \mathrm{~h}$ at room temp. affords mixtures of $\mathbf{8 a} / \mathbf{9 a}$ or $8 \mathbf{b} / \mathbf{9 b}$ without any epimerization, i.e. again 3:2 and 5:2, respectively (Scheme 1). Attempted separation of the product mixtures 8/9 either by chromatography or by preparative $\mathrm{GC}$ failed as both conditions lead to quantitative epimerization $\mathbf{8} \rightarrow \mathbf{9}$. Therefore the spectroscopic data of trans-fused cyclobutanones $\mathbf{8}$ discussed below stem from the original product mixtures and only those of the cis-fused diastereomers 9 from isolated/purified compounds. 


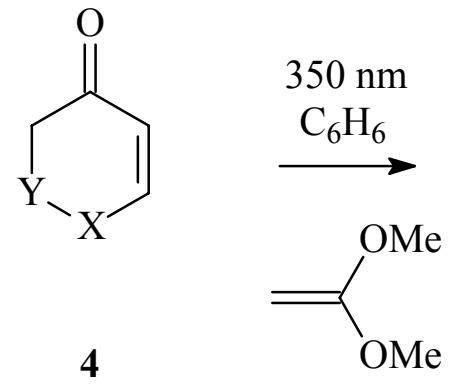

5

$4,6,7,8,9$

a: $\mathrm{X}=\mathrm{CH}_{2}, \mathrm{Y}=\mathrm{C}\left(\mathrm{CH}_{3}\right)_{2}$

b: $\mathrm{X}=\mathrm{C}\left(\mathrm{CH}_{3}\right)_{2}, \mathrm{Y}=\mathrm{CH}_{2}$

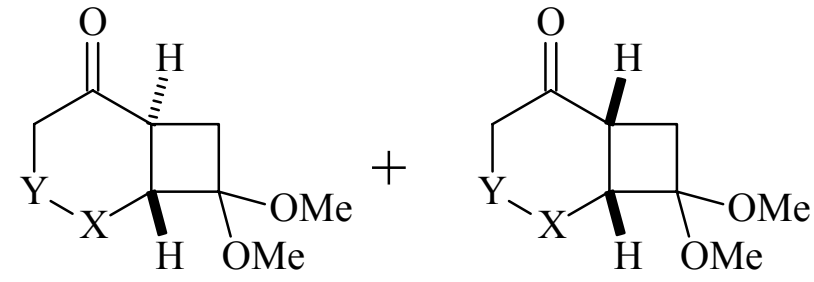

6

7

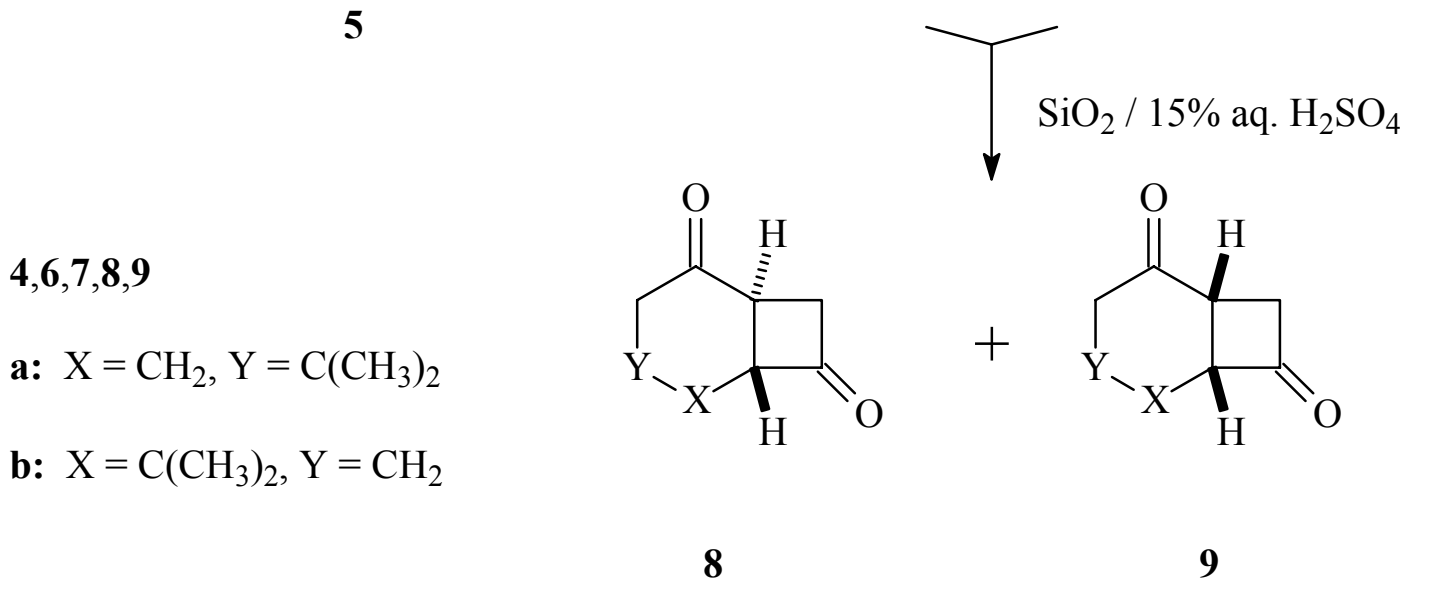

\section{Scheme 1}

The NMR-data (Fig. 2) summarizes averaged values for the (almost identical) H,H-coupling constants and ${ }^{13} \mathrm{C}$-chemical shifts of $\mathbf{8 a}, \mathbf{b}$ and $\mathbf{9 a}, \mathbf{b}$, respectively. As both the vicinal coupling constants ${ }^{3} \mathrm{~J}$ and the long range coupling constants ${ }^{4} \mathrm{~J}$ are typical for all diastereomeric pairs of bicyclo[4.2.0]octanes, it becomes evident that the best criteria to differentiate between trans- and cis-fused cyclobutanones are $a$ ) the geminal coupling constant of the methylene H-atoms adjacent to the carbonyl group ( $15 \mathrm{~Hz} v s 18 \mathrm{~Hz})$, and $b$ ) the chemical shift of the carbonyl Catom (198 vs $207 \mathrm{ppm}$ ), respectively. The former value reflects the dihedral angle $\theta$ between the $\mathrm{CH}$ bond and the p-orbital of the adjacent $\mathrm{sp}^{2}-$ hybridized $\mathrm{C}$-atom, corresponding to an expected - value near $90^{\circ}$ for (puckered) 8 and $15-25^{\circ}$ for (planar) 9 , respectively. ${ }^{9}$ In contrast, the difference in the latter - $\delta$ - values, i.e. almost $10 \mathrm{ppm}$, is remarkable, as for cyclohexanones where the ring is only slightly distorted from an ideal chair by the trans-ring fusion to a four membered ring, e.g. in $\mathbf{6 a}$ or $\mathbf{6 b}$, the carbonyl C-atoms are only shielded by $2-3 \mathrm{ppm}$ as compared to those in cis-fused $\mathbf{7 a}$ and $\mathbf{7 b}$, respectively. Increase of strain in a four-membered ring bearing a $\mathrm{sp}^{2}$-hybridized $\mathrm{C}$-atom should thus reflect on the chemical shift of this latter nucleus by becoming more similar to that of a corresponding one in a three membered ring. Unfortunately, no reliable carbonyl chemical shifts of cyclopropanones have been reported in the literature, as these compounds are not stable at room temperature. On the other side there is experimental data for the corresponding $\mathrm{sp}^{2}$-hybridized ring-C-atoms of methylenecycloalkanes, 
whith values of 131, 150, 153 and $150 \mathrm{ppm}$ for methylene-cyclopropane, -cyclobutane, cyclopentane and -cyclohexane, respectively. ${ }^{10}$ Indeed now in these compounds there is a large decrease in the $\delta$-value in going from the four- to the three membered ring, which fits with the observation mentioned above. In conclusion, we have, for the first time, identified and fully characterized spectroscopically two trans-fused bicyclo[4.2.0]octane-2,7-diones.

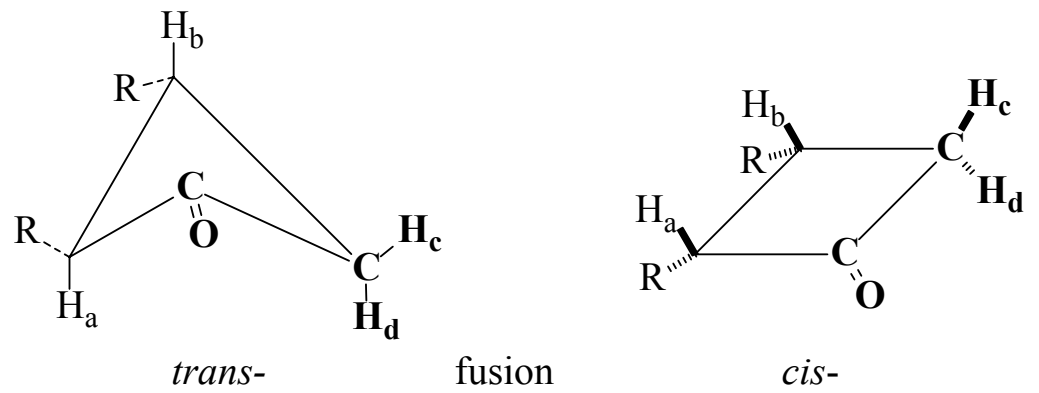

$\underline{\mathrm{H}, \mathrm{H}-\mathrm{Coupling} \text { Constants }}$

\begin{tabular}{|c|c|c|}
\hline $13.4 \mathrm{~Hz}$ & ${ }^{3} \mathrm{~J}\left(\mathrm{H}_{\mathrm{a}} \mathrm{H}_{\mathrm{b}}\right)$ & $10.4 \mathrm{~Hz}$ \\
\hline $6.5 \mathrm{~Hz}$ & ${ }^{3} \mathrm{~J}\left(\mathrm{H}_{\mathrm{b}} \mathrm{H}_{\mathrm{c}}\right)$ & $10.4 \mathrm{~Hz}$ \\
\hline $10.3 \mathrm{~Hz}$ & ${ }^{3} \mathrm{~J}\left(\mathrm{H}_{\mathrm{b}} \mathrm{H}_{\mathrm{d}}\right)$ & $5.7 \mathrm{~Hz}$ \\
\hline $15.1 \mathrm{~Hz}$ & ${ }^{2} \mathbf{J}\left(\mathbf{H}_{c} \mathbf{H}_{d}\right)$ & $18.1 \mathrm{~Hz}$ \\
\hline \multicolumn{3}{|c|}{${ }^{13} \underline{\mathrm{C}-\mathrm{Chemical} \text { Shifts }}$} \\
\hline $44 \mathrm{ppm}$ & $\mathrm{C}_{\mathrm{c}, \mathrm{d}}$ & $50 \mathrm{ppm}$ \\
\hline 198 ppm & $\mathbf{C}=\mathbf{O}$ & 207.5 ppm \\
\hline
\end{tabular}

Figure 2

\section{Experimental Section}

General Procedures. ${ }^{1} \mathrm{H}-\mathrm{NMR}$ spectra (500 MHz) and ${ }^{13} \mathrm{C}-\mathrm{NMR}$ spectra (125 MHz) - including two-dimensional plots - were recorded on a Bruker DRX 500 spectrometer. Chemical shifts $(\delta)$ are given in ppm rel. to TMS (0 ppm) as internal standard. Mass spectra were measured on a Varian MAT 311 A instrument at $70 \mathrm{eV}$. Photolyses were run in a Rayonet RPR-100 photochemical reactor equipped with (16) $350 \mathrm{~nm}$ lamps. Analytical GC was performed on a 30 m 5\% SE-30 capillary column.

Starting materials. Cyclohexenones $\mathbf{4 a}$ and $\mathbf{4 b}$ were synthesized according to the literature. ${ }^{11,12}$ 1,1-Dimethoxyethylene (5) was generously provided by Wacker Chemie AG (Munich). 
Photolyses. Ar-Degassed solns. of either $\mathbf{4 a}$ or $\mathbf{4 b}(248 \mathrm{mg}, 2 \mathrm{mmol})$ and 5 (1.76 g, $20 \mathrm{mmol})$ in benzene $(5 \mathrm{ml})$ were irradiated for $16 \mathrm{~h}$ up to total enone conversion $(\mathrm{GC})$. Irradiation of $4 \mathbf{a}$ affords $(100 \%)$ a 3:2 mixture of $\mathbf{6 a}$ and $\mathbf{7 a}$, whereas irradiation of $\mathbf{4 b}$ gives (100\%) a 5:2 mixture of $\mathbf{6 b}$ and $\mathbf{7 b}$, respectively. On attempted separation/purification by chromatography on $\mathrm{SiO}_{2}$, quantitative epimerization of 6 to 7 occurs, and therefore only the cis-fused 7,7dimethoxybicyclo[4.2.0] octan-2-ones were isolated as pure compounds, both colourless liquids.

1 $\alpha, 6 \beta-7,7-D i m e t h o x y-4,4-d i m e t h y l b i c y c l o[4.2 .0]$ octan-2-one (6a). ${ }^{1} \mathrm{H}-\mathrm{NMR}\left(\mathrm{CDCl}_{3}\right) \delta 3.27$ $(3 \mathrm{H}, \mathrm{s}), 3.19(3 \mathrm{H}, \mathrm{s}), 2.57(\mathrm{H}(1), \mathrm{dt}, J=12.8,8.5 \mathrm{~Hz}), 2.35(\mathrm{H}(6), \mathrm{dt}, J=3.3,12.8 \mathrm{~Hz}), 2.30 \&$ $1.97\left(2 \mathrm{H}(3), J_{\text {gem }}=13.5 \mathrm{~Hz}\right), 2.11 \& 2.09\left(2 \mathrm{H}(8), J_{\text {gem }}=11.5 \mathrm{~Hz}\right), 1.85 \& 1.65\left(2 \mathrm{H}(5), J_{\text {gem }}=\right.$ $12.6 \mathrm{~Hz}), 1.17(3 \mathrm{H}, \mathrm{s}), 1.03(3 \mathrm{H}, \mathrm{s}) ;{ }^{13} \mathrm{C}-\mathrm{NMR}\left(\mathrm{CDCl}_{3}\right) \delta 205.9(\mathrm{C}(2)), 103.2(\mathrm{C}(7)), 55.5$ $(\mathrm{C}(3)), 53.8(\mathrm{C}(6)), 49.2 \& 48.8\left(\mathrm{CH}_{3} \mathrm{O}\right), 44.1(\mathrm{C}(1)), 40.2(\mathrm{C}(4)), 40.1(\mathrm{C}(5)), 33.5(\mathrm{C}(8)), 32.1$ \& $29.5\left(\mathrm{CH}_{3}\right) ; \mathrm{MS} \mathrm{m} / z 181\left(\mathrm{M}^{+}-\mathrm{OCH}_{3}, 20 \%\right), 88(100 \%)$.

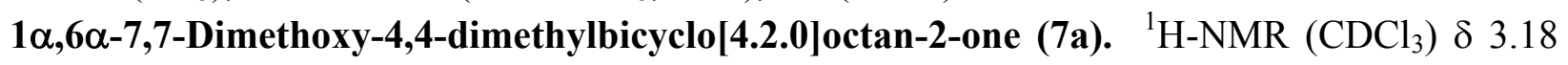
$(3 \mathrm{H}, \mathrm{s}), 3.16(3 \mathrm{H}, \mathrm{s}), 2.85(\mathrm{H}(6), \mathrm{dq}, J=3.2,9.0 \mathrm{~Hz}), 2.67(\mathrm{H}(1), \mathrm{q}, J=9.0 \mathrm{~Hz}), 2.47 \& 2.28$ $\left(2 \mathrm{H}(8), J_{\text {gem }}=12.1 \mathrm{~Hz}\right), 2.27 \& 2.25\left(2 \mathrm{H}(3), J_{\text {gem }}=16.2 \mathrm{~Hz}\right), 1.80 \& 1.63\left(2 \mathrm{H}(5), J_{\text {gem }}=13.8\right.$ $\mathrm{Hz}), 1.05(3 \mathrm{H}, \mathrm{s}), 0.94(3 \mathrm{H}, \mathrm{s}) ;{ }^{13} \mathrm{C}-\mathrm{NMR}\left(\mathrm{CDCl}_{3}\right) \delta 207.8(\mathrm{C}(2)), 101.5(\mathrm{C}(7)), 52.5(\mathrm{C}(3))$, 49.5 \& $48.9\left(\mathrm{CH}_{3} \mathrm{O}\right), 43.8(\mathrm{C}(6)), 34.7(\mathrm{C}(1)), 34.6(\mathrm{C}(4)), 34.2(\mathrm{C}(5)), 34.1(\mathrm{C}(8)), 31.1 \& 26.8$ $\left(\mathrm{CH}_{3}\right) ; \mathrm{MS} m / z 212\left(\mathrm{M}^{+}, 0.2 \%\right), 88(100 \%)$.

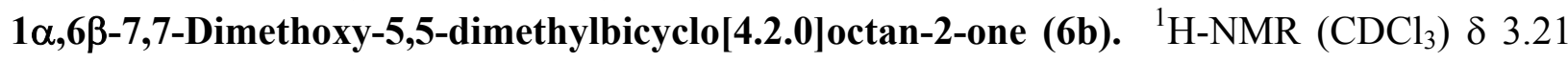
$(3 \mathrm{H}, \mathrm{s}), 3.19(3 \mathrm{H}, \mathrm{s}), 2.92(\mathrm{H}(1), \mathrm{ddd}, J=7.0,10.0,13.7 \mathrm{~Hz}), 2.40 \& 2.25\left(2 \mathrm{H}(3), J_{\text {gem }}=14.1\right.$ $\mathrm{Hz}), 2.20 \& 2.05\left(2 \mathrm{H}(8), J_{g e m}=11.4 \mathrm{~Hz}\right), 2.02(\mathrm{H}(6), \mathrm{d}, J=13.7 \mathrm{~Hz}), 1.72 \& 1.68(2 \mathrm{H}(4), J$ $\left.{ }_{\text {gem }}=12.8 \mathrm{~Hz}\right), 1.28(3 \mathrm{H}, \mathrm{s}), 1.14(3 \mathrm{H}, \mathrm{s}) ;{ }^{13} \mathrm{C}-\mathrm{NMR}\left(\mathrm{CDCl}_{3}\right) \delta 208.2(\mathrm{C}(2)), 106.9(\mathrm{C}(7)), 65.1$ (C(6)), 50.0 \& $49.8\left(\mathrm{CH}_{3} \mathrm{O}\right), 44.9(\mathrm{C}(5)), 44.1(\mathrm{C}(4)), 39.5(\mathrm{C}(1)), 36.9(\mathrm{C}(3)), 32.0(\mathrm{C}(8)), 29.8$ \& $22.1\left(\mathrm{CH}_{3}\right) ; \mathrm{MS} m / z 212\left(\mathrm{M}^{+}, 0.5 \%\right), 88(100 \%)$.

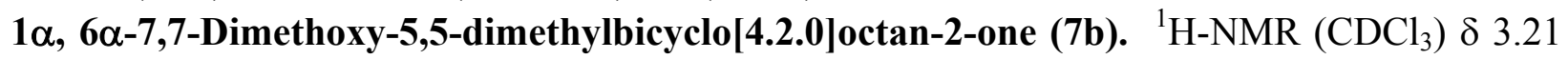
$(3 \mathrm{H}, \mathrm{s}), 3.09(3 \mathrm{H}, \mathrm{s}), 2.64(\mathrm{H}(1), \mathrm{dt}, J=3.8,9.8 \mathrm{~Hz}), 2.58(\mathrm{H}(6), \mathrm{d}, J=9.8 \mathrm{~Hz}), 2.45 \& 2.39$ $\left(2 \mathrm{H}(3), J_{\text {gem }}=16.1 \mathrm{~Hz}\right), 2.40 \& 2.05\left(2 \mathrm{H}(8), J_{\text {gem }}=12.5 \mathrm{~Hz}\right), 1.62 \& 1.50\left(2 \mathrm{H}(4), J_{\text {gem }}=12.8\right.$ $\mathrm{Hz}), 1.10(3 \mathrm{H}, \mathrm{s}), 0.99(3 \mathrm{H}, \mathrm{s}) ;{ }^{13} \mathrm{C}-\mathrm{NMR}\left(\mathrm{CDCl}_{3}\right) \delta 211.5(\mathrm{C}(2)), 101.2(\mathrm{C}(7)), 56.5(\mathrm{C}(6)), 49.8$ \& $49.2\left(\mathrm{CH}_{3} \mathrm{O}\right), 44.3(\mathrm{C}(4)), 37.2(\mathrm{C}(1)), 36.1(\mathrm{C}(5)), 35.1(\mathrm{C}(3)), 33.1(\mathrm{C}(8)), 31.2$ \& 30.1 $\left(\mathrm{CH}_{3}\right) ; \mathrm{MS} \mathrm{m} / \mathrm{z} 212\left(\mathrm{M}^{+}, 1.5 \%\right), 88(100 \%)$.

Hydrolyses. To a suspension of $150 \mathrm{mg} \mathrm{S^{ \circ }}{ }_{2}$ in $\mathrm{CH}_{2} \mathrm{Cl}_{2}(1 \mathrm{ml})$ is added $15 \mu \mathrm{l}$ of $15 \% \mathrm{H}_{2} \mathrm{SO}_{4}$. After stirring for $5 \mathrm{~min}$. a soln. of the mixture $6 / 7(21.2 \mathrm{mg}, 0.1 \mathrm{mmol})$ in $\mathrm{CH}_{2} \mathrm{Cl}_{2}(1 \mathrm{ml})$ is added, and the mixture stirred for $4 \mathrm{~h}$ at $\mathrm{rt}$. Then solid $\mathrm{NaHCO}_{3}(10 \mathrm{mg})$ is added, and stirring continued for $5 \mathrm{~min}$. The $\mathrm{SiO}_{2}$ is removed by filtration and the solvent evaporated to afford quantitatively mixtures $\mathbf{8} / \mathbf{9}$ of the same diastereomeric composition as that of the photocycloadducts, i.e. 3:2 for $\mathbf{8 a}$ and $\mathbf{9 a}$, and 5:2 for $\mathbf{8 b}$ and $\mathbf{9 b}$, respectively. On attempted separation/purification by chromatography on $\mathrm{SiO}_{2}$, again quantitative epimerization of 8 to 9 
occurs, and therefore only the cis-fused 7,7-bicyclo[4.2.0]octane-2,7-diones were isolated as pure compounds, both colourless liquids.

1 $\alpha, 6 \beta-4,4-D i m e t h y l b i c y c l o[4.2 .0]$ octane-2,7-dione (8a). ${ }^{1} \mathrm{H}-\mathrm{NMR}\left(\mathrm{CDCl}_{3}\right) \delta 3.31(\mathrm{H}(6)$, ddd, $J=3.8,12.8,13.8 \mathrm{~Hz}), 3.30 \& 2.60\left(2 \mathrm{H}(8), J_{\text {gem }}=15.1 \mathrm{~Hz}\right), 3.14(\mathrm{H}(6), \mathrm{ddd}, J=6.6,10.3$, $13.8 \mathrm{~Hz}), 2.45 \& 2.15\left(2 \mathrm{H}(3), J_{\text {gem }}=13.3 \mathrm{~Hz}\right), 1.95 \& 1.80\left(2 \mathrm{H}(5), J_{\text {gem }}=12.8 \mathrm{~Hz}\right), 1.17(3 \mathrm{H}$, s), $1.06(3 \mathrm{H}, \mathrm{s}) ;{ }^{13} \mathrm{C}-\mathrm{NMR}\left(\mathrm{CDCl}_{3}\right) \delta 204.8(\mathrm{C}(2)), 198.2(\mathrm{C}(7)), 66.5(\mathrm{C}(6)), 56.2(\mathrm{C}(3)), 44.2$ $(\mathrm{C}(8)), 41.6(\mathrm{C}(1)), 41.5(\mathrm{C}(4)), 39.5(\mathrm{C}(5)), 31.92 \& 27.7\left(\mathrm{CH}_{3}\right)$.

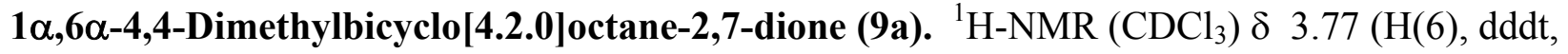
$J=4.5,9.3,10.8,3.0 \mathrm{~Hz}), 3.42 \& 3.25\left(2 \mathrm{H}(8), J_{\text {gem }}=18.1 \mathrm{~Hz}\right), 3.06(\mathrm{H}(1), \mathrm{dt}, J=5.5,10.8 \mathrm{~Hz})$, $2.32 \& 2.27\left(2 \mathrm{H}(3), J_{\text {gem }}=15.1 \mathrm{~Hz}\right), 1.90 \& 1.70\left(2 \mathrm{H}(5), J_{\text {gem }}=14.0 \mathrm{~Hz}\right), \quad 1.05(3 \mathrm{H}, \mathrm{s}), 0.90$ $(3 \mathrm{H}, \mathrm{s}) ;{ }^{13} \mathrm{C}-\mathrm{NMR}\left(\mathrm{CDCl}_{3}\right) \delta 211.5(\mathrm{C}(2)), 208.5(\mathrm{C}(7)), 58.1(\mathrm{C}(6)), 54.1(\mathrm{C}(4)), 52.2(\mathrm{C}(3))$, $52.1(\mathrm{C}(8)), 36.2(\mathrm{C}(5)), 34.1(\mathrm{C}(1)), 30.3 \& 30.1\left(\mathrm{CH}_{3}\right)$; $\mathrm{MS} \mathrm{m} / \mathrm{z} 166\left(\mathrm{M}^{+}, 30 \%\right), 68(100 \%)$.

1 $\alpha, 6 \beta-5,5-D i m e t h y l b i c y c l o[4.2 .0]$ octane-2,7-dione (8b). ${ }^{1} \mathrm{H}-\mathrm{NMR}\left(\mathrm{CDCl}_{3}\right) \delta 3.23 \& 2.60$ $\left(2 \mathrm{H}(8), J_{\text {gem }}=15.0 \mathrm{~Hz}\right), 3.01(\mathrm{H}(6)$, d. $J=13.0 \mathrm{~Hz}), 3.00(\mathrm{H}(1)$, ddd, $J=6.6,10.3,13.0 \mathrm{~Hz})$, $2.50 \& 2.32\left(2 \mathrm{H}(3), J_{\text {gem }}=14.5 \mathrm{~Hz}\right), 1.80 \& 1.75\left(2 \mathrm{H}(4), J_{\text {gem }}=12.8 \mathrm{~Hz}\right), \quad 1.25(3 \mathrm{H}, \mathrm{s}), 1.16$ $(3 \mathrm{H}, \mathrm{s}) ;{ }^{13} \mathrm{C}-\mathrm{NMR}\left(\mathrm{CDCl}_{3}\right) \delta 206.5(\mathrm{C}(2)), 198.2(\mathrm{C}(7)), 76.1(\mathrm{C}(6)), 43.3(\mathrm{C}(8)), 43.2(\mathrm{C}(4))$, $38.3(\mathrm{C}(3)), 35.5(\mathrm{C}(1)), 34.5(\mathrm{C}(5)), 28.72 \& 28.2\left(\mathrm{CH}_{3}\right)$.

1 $\alpha, 6 \alpha-5,5-D i m e t h y l b i c y c l o[4.2 .0]$ octane-2,7-dione (9b). ${ }^{1} \mathrm{H}-\mathrm{NMR}\left(\mathrm{CDCl}_{3}\right) \delta 3.38(\mathrm{H}(6), \mathrm{dt}, J$ $=10.0,3.0 \mathrm{~Hz}), 3.34 \& 3.04\left(2 \mathrm{H}(8), J_{\text {gem }}=18.0 \mathrm{~Hz}\right), 3.14(\mathrm{H}(1), \mathrm{dt}, J=6.0,10.0 \mathrm{~Hz}), 2.53 \&$ $2.51\left(2 \mathrm{H}(3), J_{\text {gem }}=15.1 \mathrm{~Hz}\right), 1.88 \& 1.70\left(2 \mathrm{H}(5), J_{\text {gem }}=14.0 \mathrm{~Hz}\right), 1.17(3 \mathrm{H}, \mathrm{s}), 1.03(3 \mathrm{H}, \mathrm{s}) ;$

${ }^{13} \mathrm{C}-\mathrm{NMR}\left(\mathrm{CDCl}_{3}\right) \delta 211.5(\mathrm{C}(2)), 207.5(\mathrm{C}(7)), 72.5(\mathrm{C}(6)), 50.2(\mathrm{C}(8)), 36.1(\mathrm{C}(1)), 35.5$ (C(3)), $34.5(\mathrm{C}(5)), 33.8(\mathrm{C}(4)), 26.6 \& 26.5\left(\mathrm{CH}_{3}\right)$; $\mathrm{MS} \mathrm{m} / z 166\left(\mathrm{M}^{+}, 2 \%\right), 82(100 \%)$.

\section{References and Notes}

1. Corey, E. J.; Bass, J. D.; LeMahieu, R.; Mitra, R. B. J Am. Chem. Soc. 1964, 86, 5570.

2. Margaretha, P. In Molecular and Supramolecular Photochemistry, Vol. 12; Griesbeck, A. G.; Mattay, J., Eds.; Marcel Dekker: New York, 2005; pp 211-238, and refs. cit. therein.

3. Spelbos, A.; Mijlhoff, F. C.; Bakker, W. H.; Baden, R.; Van Den Enden, L. J. Molec. Struct. 1977, 38, 155.

4. Schmidt, K.; Kopf, J.; Margaretha, P. Helv. Chim. Acta 2006, 89, 1927.

5. Novak, I.; Harrison, L. J.; Li, W.; Kovac, B. J. Phys. Chem. A 2007, 111, 2619.

6. Lenz, G. R. J. Chem. Soc. Perkin Trans. 1 1984, 2397.

7. Schmidt, K.; Kopf, J.; Margaretha, P. Helv. Chim. Acta 2007, 90, 1667.

8. Huet, F.; Lechevallier, A.; Pellet, M.; Conia, J. Synthesis 1978, 63.

9. Cookson, R. C.; Crabb, T. A.; Frankel, J. J.; Hudec, J. Tetrahedron 1966, Suppl. 7, 355.

10. Hung, J. T.; Yang, S. W.; Gray, G. M.; Lammertsma, K. J. Org. Chem. 1993, 58, 6786.

11. Wawrenczyk, C.; Lochynski, S. Monatsh. Chem. 1981, 116, 99.

12. Flaugh, M. E.; Crowell, T. A.; Farlow, D. S. J. Org. Chem. 1980, 45, 5399. 